\title{
Controlling the Biexciton-Exciton Cascade Kinetics in a Quantum Dot via Coupling to a Microcavity Optical Mode
}

\author{
G. $\mathrm{SezK}^{a}$, D.N. Krizhanovski ${ }^{b}$, V.D. Kulakovski ${ }^{c}$, S. Reitzenstein ${ }^{d}$ And M. Kamp $^{e}$ \\ ${ }^{a}$ Laboratory for Optical Spectroscopy of Nanostructures, Department of Experimental Physics, \\ Faculty of Fundamental Problems of Technology, Wrocław University of Technology, \\ Wybrzeże Wyspiańskiego 27, 50-370 Wrocław, Poland \\ ${ }^{b}$ Department of Physics and Astronomy, University of Sheffield, Sheffield S3 7RH, United Kingdom \\ ${ }^{c}$ Institute of Solid State Physics, Russian Academy of Sciences, Chernogolovka 142432, Russia \\ ${ }^{d}$ Institute of Solid State Physics, Berlin University of Technology, Berlin 10623, Germany \\ eTechnische Physik, University of Würzburg and Wilhelm-Conrad-Röntgen-Research Center \\ for Complex Material Systems, Am Hubland, Würzburg 97074, Germany
}

\begin{abstract}
The luminescence of single and two exciton states in a quantum dot embedded in the active layer of a micropillar cavity has been investigated. Temperature tuning has been used to bring the energy states of the quantum dot and the cavity into resonance. Studying the resonance behavior of the exciton and biexciton transitions with cavity mode revealed a similar Purcell effect for both lines. The cavity-induced changes of the respective radiative lifetimes have been shown to allow for controlling the ratio between the single and two exciton occupation and their relative emission rates in a single quantum dot.
\end{abstract}

DOI: 10.12693/APhysPolA.129.A-44

PACS: 78.67.Hc, 71.35.--y, 78.55.-m, 42.50.Pq

\section{Introduction}

Almost seventy years ago Purcell suggested the idea to control spontaneous emission (SE) rate of a quasimonochromatic dipole by using a cavity [1]. This effect was experimentally observed in the 80's in studies of the emission of atoms passed through a cavity: the atom exhibited either enhancement or inhibition of the SE rate, depending on its position at an antinode or node of the cavity mode (the Purcell effect) [2-4]. The enhancement for an atom-like emitter placed into the antinode of the cavity mode is described by the Purcell factor

$$
F_{\mathrm{P}}=\left(3 / 4 \pi^{2}\right)(\lambda / n)^{3} Q / V_{\mathrm{m}},
$$

where $\lambda$ is the resonant wavelength and $n$ is the refractive index of the cavity with effective mode volume $V_{\mathrm{m}}$ and cavity quality factor Q. In a semiconductor system, the electron-hole pair captured by a semiconductor quantum dot $(\mathrm{QD})$ can be treated as an atom-like emitter with a discrete set of available energy states. Progress in microfabrication technologies has allowed a realization of quantum electrodynamics (QED) experiments in solids utilizing semiconductor cavities containing QDs. Although the strong coupling regime has already been demonstrated on the single photon - single exciton (electron) level in many systems [5-7] (i.e. when the escape time of photons out of the cavity is comparable with radiative time of $\mathrm{QD}$ transitions and the process of SE becomes reversible, resulting in the Rabi splitting of resonant energies and formation of quasi 0D polaritons), the weak coupling QED is still explored mainly due to significant application potential. It concerns mainly quantum information processing and quantum cryptography [8-11], including many demonstrations of high speed and efficient single photon sources [12-15] or entangled photon pairs [16]. Starting from the pioneering work of Gerard [17] showing the enhancement of the SE rate of a QD ensemble placed in a 3D-cavity, the Purcell effect was demonstrated both in optical experiments on single QD placed either into micropillar or photonic-crystal defect cavity [18-20] and in measurements of photocurrent generated in a QD coupled to a microcavity mode $[21,22]$. In this paper we studied photoluminescence (PL) from a single micropillar cavity (MPC) with a set of single QDs in the active layer, where the change of temperature was used to tune single QD emission lines into resonance with the cavity mode. The on- and off-resonance excitation power dependence of the PL intensities has been found to be different for the exciton and biexciton emission. However, both the transitions demonstrate similar Purcell effect.

\section{Experimental}

Pillars with a circular cross-section have been processed through reactive ion-etching of a GaAs/AlAs planar microcavity structure grown by molecular beam epitaxy. The Bragg reflectors are composed of 20 (23) repetitions of $\lambda / 4 \mathrm{GaAs} / \mathrm{AlAs}(69 \mathrm{~nm} / 82 \mathrm{~nm}$ ) layers in the top (bottom) mirrors. The $\lambda$ GaAs active layer contains an array of self-assembled $\operatorname{In}_{0.6} \mathrm{Ga}_{0.4} \mathrm{As}$ QDs (density $\approx 10^{10} \mathrm{~cm}^{-2}$ ) placed at the antinode of the on-axis resonant fundamental mode of the planar cavity.

The photoluminescence was measured using a highresolution one meter focal length double-monochromator with a liquid nitrogen cooled Si CCD as a detector. We used a high numerical aperture microscope objective to focus the excitation laser with a wavelength of $532 \mathrm{~nm}$ (second harmonic of a cw Nd:YAG laser) to a spot with 
a diameter of about $3 \mu \mathrm{m}$ and to collect the emitted radiation. The QD emission of an unpatterned sample is located in the spectral range of $1.36-1.39 \mathrm{eV}$. The planar microcavity was grown so that the resonant energy of the photon mode is located slightly below the maximum of the QD emission. In circularly shaped micropillar prepared from the planar cavity the combination of waveguiding by the sidewalls and of the longitudinal confinement by the distributed Bragg reflectors (DBRs) results in a set of $3 \mathrm{D}$ confined modes. In this paper we concentrated on the study of the micropillars with diameter $d_{\mathrm{C}} \approx 1.4 \mu \mathrm{m}$ and $Q \approx 4500$, whereas the energy of ground state photon mode is expected to be around $1.3-1.32 \mathrm{eV}$. In this case, only a small number of QDs in the pillar has transition energies in the range of the cavity mode, which allowed us to study the effect of cavity on the emission from various QDs independently of each other.

\section{Results and discussion}

Figure 1a shows exemplary $\mu \mathrm{PL}$ spectra from a micropillar with a core diameter of $1.4 \mu \mathrm{m}$ recorded at $5 \mathrm{~K}$ and for two excitation powers $P=10$ and $70 \mu \mathrm{W}$ (measured outside the cryostat). The emission was collected along the normal to the DBRs' surface in a solid angle of approximately $0.045 \pi$. The spectra consist of a number of narrow lines with the full width at half maximum $\gamma$ smaller than $0.06 \mathrm{meV}$ corresponding to the emission of single QDs and a relatively broad line at $1.3029 \mathrm{eV}$ with the $\gamma_{\mathrm{C}} \approx 0.29 \mathrm{meV}$ corresponding to the cavity (C) mode. Increase of the excitation power density results in the appearance of additional narrow lines, which can be related to the occupation of the dot with a second exciton. In particular, we are interested in the line that appears in the high excitation spectrum slightly above the cavity mode, at $1.304 \mathrm{eV}$, and can be tuned to the cavity mode with a reasonable increase of the sample temperature up to about $30 \mathrm{~K}$. This line appears at $0.8 \mathrm{meV}$ below the line at $1.3048 \mathrm{eV}$ in a low excitation spectrum, i.e. in a range typical for the biexciton binding energy in self-assembled $\operatorname{In}(\mathrm{Ga})$ As QDs.

To prove such an origin of these lines we have measured the excitation power $-P$-dependence when both are off resonance with the cavity mode - shown in Fig. 1b. A common behavior has been obtained where the exciton and biexciton lines intensity, $I_{\mathrm{X}}$ and $I_{\mathrm{XX}}$, depend linearly and quadratically, respectively, in the low excitation regime, and then saturate accordingly. The saturation and decrease of the single exciton emission $I_{\mathrm{X}}$ with further increase in $P$ coincides with the deviation of the dependence of $I_{\mathrm{XX}}$ from the square one. However, the sum of intensities $I_{\mathrm{X}}+I_{\mathrm{XX}}$ continues to be linear in $P$ in a wide range of $P<80 \mu \mathrm{W}$ until the excitation rate becomes similar to the recombination rate for XX states. Thus, both emission lines (X and XX) can be considered to originate from the same QD.

Further, we have considered the behavior of the exciton and biexciton emission when either the exciton or biexciton transition energy is tuned into resonance with the
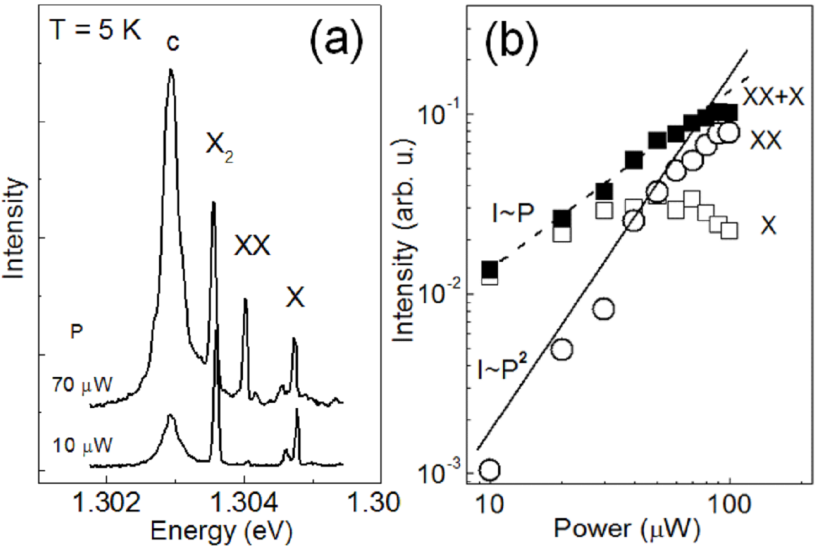

Fig. 1. (a) PL spectra from $1.4 \mu \mathrm{m}$ diameter micropillar at two excitation densities at $5 \mathrm{~K}$, (b) Power dependence of the intensities of single (X) and two (XX) exciton states from the same QD. The solid dots show the sum of exciton and biexciton emission intensities from one dot.

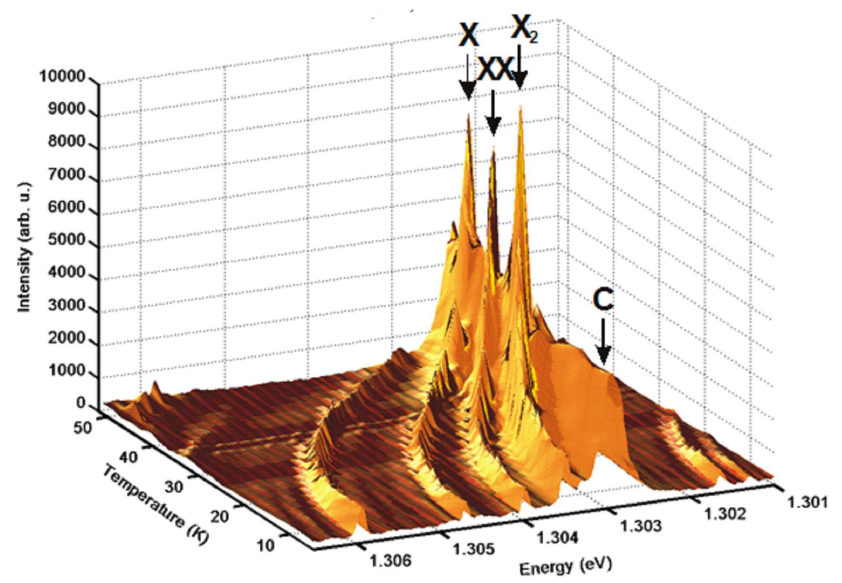

Fig. 2. Set of PL spectra from the micropillar in a range of $5-50 \mathrm{~K} ; \mathrm{X}_{2}$ labels an emission line from another dot.

cavity mode. Figure 2 displays PL spectra in the temperature range of $5-50 \mathrm{~K}$. Both lines $\mathrm{X}$ and $\mathrm{XX}$ shift in this temperature range to lower energies by about $3 \mathrm{meV}$, while the $\mathrm{C}$ line shift is about 5 times smaller $(\approx 0.6 \mathrm{meV})$, which is related to various thermal coefficients of the QD material energy gap and the refractive index of the cavity, respectively [16]. It is seen from Fig. 3 which shows the energy positions of the respective peaks, that the lines XX and $\mathrm{X}$ get into the resonance with the $\mathrm{C}$ mode at $T=33.8$ and $44.9 \mathrm{~K}$, respectively. No line splitting is observed in both, Fig. 2 and Fig. 3, i.e. when the X (XX) transition is on the resonance with cavity mode, which indicates that the system is in a weak coupling regime.

Tracing the PL intensity of QD lines as they are tuned by temperature across the $\mathrm{C}$ mode at high excitation power $(P=100 \mu \mathrm{W})$, we have plotted in Fig. 4 the dependences of exciton (X) and biexciton (XX) emission intensities versus detuning of $\mathrm{QD}$ and cavity mode $\Delta$. 


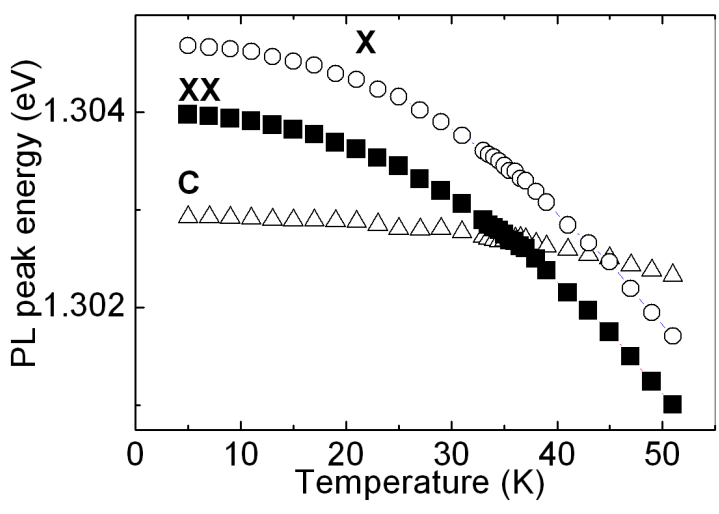

Fig. 3. Temperature dependence of cavity (C), exciton $(\mathrm{X})$ and biexciton (XX) transition energies.

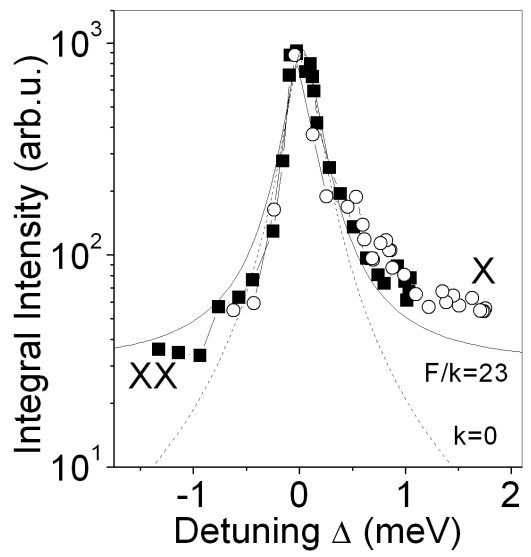

Fig. 4. The intensities of exciton (open circles) and biexciton (solid squares) emission versus QD-cavity mode detuning $\Delta$. The theoretical fit is shown by the thin solid line. The dashed lines show the intensity calculated without contribution from leaky modes.

( $\Delta$ equals to $E_{\mathrm{XX}}-E_{\mathrm{C}}$ and $E_{\mathrm{X}}-E_{\mathrm{C}}$ for $\mathrm{X}$ and $\mathrm{XX}$ lines, respectively.) Figure 4 shows that both $I_{\mathrm{XX}}$ and $I_{\mathrm{X}}$ demonstrate the same maximal magnitude of enhancement due to the Purcell effect at zero detuning $(\Delta=0)$. The QD emission registered by the detector is collected from the top of the micropillar (through the DBR) and partly from the sidewalls of micropillar (leaky modes). In this case, the detected emission intensity is

$$
I_{i} \sim w_{i}\left(1 / \tau_{i}^{*}+k / \tau_{i}^{* *}\right),
$$

where $w_{i}$ is the probability of the QD occupation by one $(i=\mathrm{X})$ or two $(i=\mathrm{XX})$ excitons, $1 / \tau_{i}^{*}$ and $1 / \tau_{i}^{* *}$ are the radiative decays of the corresponding state through the DBR mirrors and sidewalls (leaky modes), respectively, and the coefficient $k<1$ reflects the fact that only part of sidewall emission is collected in the experiment. In the weak coupling regime $1 / \tau_{i}^{*}$ can be expressed as

$$
\frac{1}{\tau_{i}^{*}} \sim F_{i} \frac{\left(\gamma_{\mathrm{c}} / 2\right)^{2}}{\left(\Delta^{2}+\left(\gamma_{\mathrm{c}} / 2\right)^{2}\right)} \frac{1}{\tau_{0, i}} .
$$

Here $1 / \tau_{0, i}$ is the QD SE rate in bulk, $1 / \tau^{* *} \approx 0.8 / \tau_{0, i}$ is the $\mathrm{SE}$ rate of the $\mathrm{QD}$ in a micropillar to the leaky modes $[5], \gamma_{c}$ is the full width at half maximum of the
C line when the cavity mode is off the resonance with any QD oscillator, and $F_{i}$ is the Purcell factor.

It follows from kinetic equations that $w_{\mathrm{X}} \sim 1 / \tau_{\mathrm{X}}$ until the generation rate $g<1 / \tau_{\mathrm{X}}$. Thereby, the Purcell effect is well pronounced in the QD emission intensity only at high excitation densities when the generation rate $g \gg 1 / \tau$ and $w_{\mathrm{X}}$ depends weakly on $\tau_{\mathrm{X}}$. Figure 4 shows the fit of the experimental dependences $I_{\mathrm{X}, \mathrm{Xx}}(P)$ recorded in the saturation regime $(P=100 \mu \mathrm{W})$ with formulae (2) and (3). Here, we have taken $w_{\mathrm{X}, \mathrm{XX}}=$ const and used only two adjustable parameters, the intensity at the maximum and the ratio $F_{i} / k$. To find out about the effect of the cavity, we have plotted in Fig. 4 the emission intensity calculated without taking into account the emission from the sidewalls (dashed line). The latter shows that the changes in the emission intensity in the range of cavity resonance are predominantly determined by the Purcell effect due to coupling of QD to the cavity mode. The best fit in Fig. 4 for the exciton and biexciton emission is obtained at a similar ratio of $F_{i} / k$ equal approximately to 23 . In order to derive $F_{i}$ an estimation of $k$ needs to be made. In the solid angle corresponding to the used experimental configuration the relative fraction of the collected leaky modes emission can be very roughly estimated to about $0.25-0.3$. This results in the estimation of the Purcell factor in the range of 5.7-6.9.

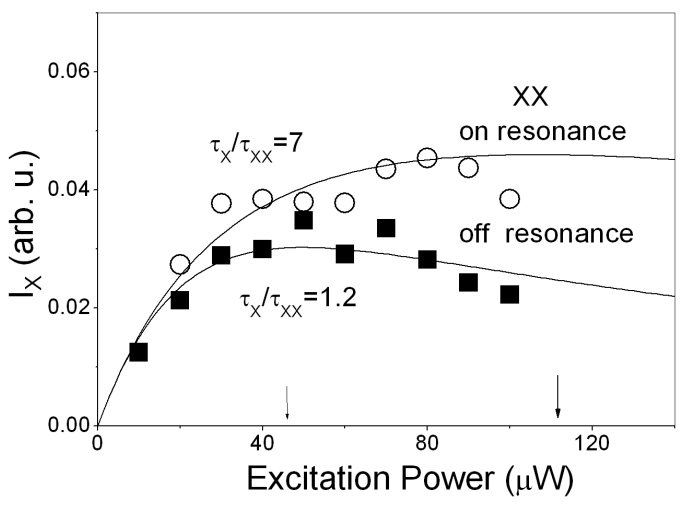

Fig. 5. The intensity of exciton transition as function of excitation power measured at $27 \mathrm{~K}$ and $34 \mathrm{~K}$ when the XX line on (circles) and off (triangles) resonance with the cavity mode. The lines show the theoretical simulations of exciton emission intensity for $\tau_{\mathrm{X}} / \tau_{\mathrm{XX}}=$ 1.2 (XX off resonance) and 7 (XX on resonance).

The possibility to change the lifetime of either one or two exciton state in the QD opens the possibility to control the QD occupation with one or two excitons at fixed excitation density. To reveal this effect we have measured the intensity of the $\mathrm{X}$ line as function of excitation power at two temperatures, $27 \mathrm{~K}$ and $34 \mathrm{~K}$, when the exciton transition is well off the resonance, whereas the XX line is off (squares) and on (circles) the resonance with the cavity mode, respectively. The dependences are shown in Fig. 5. In the case when the biexciton transition is off the resonance with the cavity mode, the exciton emission saturates at $P \approx 35-50 \mu \mathrm{W}$ and then decreases 
because of increasing occupation probability of the QD with two excitons. Shifting the biexciton transition to the resonance with cavity mode decreases its lifetime and thus increases the time of the QD occupation with the exciton only. That leads to an increase of single QD exciton emission and significant shift of the $\mathrm{X}$ line saturation to the higher excitation densities. Such a behavior can be described on the base of kinetic equations for the exciton and biexciton transitions

$$
\begin{gathered}
\mathrm{d} w_{\mathrm{X}} / \mathrm{d} t=-w_{\mathrm{X}} / \tau_{\mathrm{X}}+w_{\mathrm{XX}} / \tau_{\mathrm{XX}} \\
+g\left(1-2 w_{\mathrm{X}}-w_{\mathrm{XX}}\right), \\
\mathrm{d} w_{\mathrm{XX}} / \mathrm{d} t=-w_{\mathrm{XX}} / \tau_{\mathrm{XX}}+g w_{\mathrm{X}},
\end{gathered}
$$

where $w_{\mathrm{X}}$ and $w_{\mathrm{XX}}$ are the probabilities of the QD occupation with one and two excitons, respectively. In the stationary case one can obtain for the single exciton emission intensity that

$$
I_{\mathrm{X}} \sim \frac{w_{\mathrm{X}}}{\tau_{\mathrm{X}}} \sim \frac{g}{1+g \tau_{\mathrm{X}}+g^{2} \tau_{\mathrm{X}} \tau_{\mathrm{XX}}} .
$$

The maximum of $I_{\mathrm{X}}$ is equal to $I_{\mathrm{X}}^{*}=1 /\left(2 \sqrt{\tau_{\mathrm{X}} \tau_{\mathrm{XX}}}+\tau_{\mathrm{X}}\right)$ and occurs at $g^{*}=1 / \sqrt{\tau_{\mathrm{X}} \tau_{\mathrm{XX}}}$. That means that a 10 fold decrease in $\tau_{\mathrm{XX}}$ leads to a twofold increase in $I_{\mathrm{X}}^{*}$ and a threefold increase in $g^{*}$. Using formula (6) we have fitted the power dependences of the intensity of QD exciton transition shown in Fig. 5 for the cases when the XX line is off and on resonance with cavity mode, respectively, using a single adjustable parameter $\tau_{\mathrm{XX}} / \tau_{\mathrm{X}}$. The change in $\tau_{\mathrm{X}}$ is negligible because the exciton is well off resonance in both cases. As it is seen from the figure the experimental curves are well described under condition that the biexciton lifetime decreases about 6 times when it is on resonance with the cavity mode.

\section{Conclusion}

In conclusion, photoluminescence from a single pillar microcavity with QDs in the active layer has been measured in the temperature range of $5-50 \mathrm{~K}$. The increase in the spontaneous emission of exciton and biexciton in the same QD due to the Purcell effect has been found to be similar. The power dependence of the exciton emission changes significantly when the biexciton transition is tuned into resonance with the cavity. The variation of the exciton (biexciton) lifetime caused by the Purcell effect has been shown to allow for controlling the ratio between the single and two exciton occupation and the entire kinetics of the exciton-biexciton cascade in a single QD.

\section{Acknowledgments}

The work has partly been supported by project No. 2011/02/A/ST3/00152 of National Science Centre of Poland.

\section{References}

[1] E.M. Purcell, Phys. Rev. 69, 681 (1946).

[2] P. Goy, J.M. Raimond, M. Cross, S. Haroche, Phys. Rev. Lett. 50, 1903 (1983).

[3] G. Gabrielse, H. Dehmelt, Phys. Rev. Lett. 55, 67 (1985).
[4] R.G. Hulet, E.S. Hilfer, D. Kleppner, Phys. Rev. Lett. 55, 2137 (1985).

[5] J.P. Reithmaier, G. Sęk, A. Löffler, C. Hofmann, S. Kuhn, S. Reitzenstein, L.V. Keldysh, V.D. Kulakovskii, T.L. Reinecke, A. Forchel, Nature 432, 197 (2004).

[6] T. Yoshie, A. Scherer, J. Hendrickson, G. Khitrova, H. Gibbs, G. Rupper, C. Ell, O. Shchekin, D. Deppe, Nature 432, 200 (2004).

[7] K. Hennessy, A. Badolato, M. Winger, D. Gerace, M. Atatüre, S. Gulde, S. Fält, E.L. Hu, A. Imamoglu, Nature 445, 896 (2007).

[8] J.P. Vasco, P.S.S. Guimarães, D. Gerace, Phys. Rev. B 90, 155436 (2014).

[9] A. Imamoglu, D.D. Awschalom, G. Burkard, D.P. DiVincenzo, D. Loss, M. Sherwin, A. Small, Phys. Rev. Lett. 83, 4204 (1999).

[10] Z. Yuan, B.E. Kardynal, R.M. Stevenson, A.J. Shields, C.J. Lobo, K. Cooper, N.S. Beattie, D.A. Ritchie, M. Pepper, Science 295, 102 (2002).

[11] P. Michler, A. Kiraz, C. Becher, W.V. Schoenfeld, P.M. Petroff, L. Zhang, E. Hu, A. Imamoglu, Science 290, 2282 (2000).

[12] T. Jakubczyk, H. Franke, T. Smoleński, M. Ściesiek, W. Pacuski, A. Golnik, R. Schmidt-Grund, M. Grundmann, C. Kruse, D. Hommel, P. Kossacki, ACS Nano 8, 9970 (2014).

[13] C. Kistner, K. Morgener, S. Reitzenstein, C. Schneider, S. Höfling, L. Worschech, A. Forchel, P. Yao, S. Hughes, Appl. Phys. Lett. 96, 221102 (2010).

[14] A.K. Nowak, S.L. Portalupi, V. Giesz, O. Gazzano, C. Dal Savio, P.-F. Braun, K. Karrai, C. Arnold, L. Lanco, I. Agnes, A. Lemaître, P. Senellart, $\mathrm{Na}$ ture Commun. 5, 3240 (2013).

[15] T. Heindel, C. Schneider, M. Lermer, S.H. Kwon, T. Braun, S. Reitzenstein, S. Höfling, M. Kamp, A. Forchel, Appl. Phys. Lett. 96, 011107 (2010).

[16] A. Dousse, J. Suffczyński, A. Beveratos, O. Krebs, A. Lemaître, I. Sagnes, J. Bloch, P. Voisin, P. Senellart, Nature 466, 217 (2010).

[17] J.M. Gerard, B. Sermage, B. Gayral, B. Legrand, E. Costard, V. Thierry-Mieg, Phys. Rev. Lett. 81 , 1110 (1998).

[18] G.S. Solomon, M. Pelton, Y. Yamomoto, Phys. Rev. Lett. 86, 3903 (2001).

[19] T.D. Happ, I.I. Tartakovskii, V.D. Kulakovskii, J.P. Reithmaier, M. Kamp, A. Forchel, Phys. Rev. B 66, 041303 (2002).

[20] A. Musiał, C. Hopfmann, T. Heindel, C. Gies, M. Florian, H.A.M. Leymann, A. Foerster, C. Schneider, F. Jahnke, S. Höfling, M. Kamp, S. Reitzenstein, Phys. Rev. B 91, 205310 (2015).

[21] Yueh-Nan Chen, Der-San Chuu, Phys. Rev. B 66 165316 (2002).

[22] P. Gold, M. Gschrey, C. Schneider, S. Höfling, A. Forchel, M. Kamp, S. Reitzenstein, Phys. Rev. B 86, 161301(R) (2012). 